CULTURA, LENGUAJE Y REPRESENTACIÓN / CULTURE, LANGUAGE AND REPRESENTATION • ISSN 1697-7750 • VOL. XV \2016, PP. 35-49 REVISTA DE ESTUDIOS CULTURALES DE LA UNIVERSITAT JAUME I / CULTURAL STUDIES JOURNAL OF UNIVERSITAT JAUME I

DOI: HTTP://DX.DOI.ORG/10.6035/CLR.2016.15.3

\title{
Cuando despertaron, la democracia todavía estaba alli*
}

\author{
Upon awakening, democracy was still there
}

\author{
ANTONio alaminos CHICA \\ CLEMENTE PENALVA VERDÚ \\ UNIVERSITAT D'ALACANT
}

Recibido: 11-10-2014

Aceptado: 12-03-2015

RESUMEN: Las formas de la protesta social en España, entendidas como prácticas de participación política, contienen elementos que remiten a la recuperación y la práctica por parte de la ciudadanía de lo que consideran como verdadera democracia. En un contexto de crisis económica y social, frente a la ausencia de soberanía de unos gobiernos sometidos que prefieren rescatar a los bancos antes que a la ciudadanía, la sociedad española intenta rescatar la democracia. En España, tras caer desde las cotas más altas del consumo al enorme socavón de desempleo y pobreza, y tras los sucesos del $15 \mathrm{M}$ y el movimiento asociado surgido a la sazón, se puede observar cómo la protesta social ha pasado de la reivindicación de una democracia real como objeto difuso y multidimensional a la reivindicación de recuperación de las facetas concretas del estado de bienestar (principal logro de la democracia representativa liberal) que han sido canceladas por las políticas económicas de austeridad. El análisis de las formas de protesta (movilizaciones, manifiestos) nos lleva al encuentro con dos tipos de participación política: $a$ ) un tipo de participación política que supone una continuación 15M (con un mayor peso del componente expresivo, emocional de la acción política) que tiene como marco de producción de discursos y prácticas la idea de transformación social hacia otro modelo productivo y medioambiental, de representación política, de cambio cultural; y $b$ ) un tipo de participación política (con un mayor peso del componente racional, cognitivo) que tiene como marco de producción de discursos y prácticas la resistencia (retorno, adaptación), frente a la mutación del Estado. Como ejemplos del primer modelo tendríamos las convocatorias de «rodea el congreso», «marchas de la dignidad» y «escraches»; como ejemplos del segundo se identifican

* Este artículo se ha desarrollado en el contexto de un proyecto europeo del Programa Marie Curie, IRSES2, dirigido por el profesor Gary Spolander y coordinado en España por la profesora María Asunción Martínez Roman. REF. FP7-PEOPLE-2012-IRSES. CIVIL-SW. Civil Engagement in Social Work: Developing Global Models. 
las «mareas ciudadanas». Entre medias se pueden encontrar las plataformas de ayuda a las víctimas de la crisis (PAH).

Palabras clave: movimientos sociales, cultura de consumo, protesta, participación política, sociedad civil.

ABSTRACT: The forms of social protest in Spain, understood as practices of political participation, contain elements referring to recovery and practice by the citizens of what they see as «true democracy». In a context of economic and social crisis, facing the absence of sovereignty of subjected governments that prefer rescue banks than citizens; Spanish society tries to rescue democracy. In Spain, after falling from the highest levels of consumption to huge sinkhole of unemployment and poverty; and after the events of $15 \mathrm{M}$ and the associated movement that emerged at the time, it can be seen how social protest has passed from the claim for real democracy as diffuse and multidimensional object, to the vindication of recovery of specific aspects of the welfare state (main achievement of liberal representative democracy) that have been canceled by the economic policies of austerity. The analysis of the forms of political protest (demonstrations, manifestos) leads us to encounter two types of political participation: a) a type of political participation which is a continuation of $15 \mathrm{M}$ (with a greater weight expressive and emotionally of political action) and has as a frame of speech production and practices the idea of social transformation to another productive and environmental model of political representation, cultural change; and b) a type of political participation, weighing more rational, cognitive component, and has as a frame of discourse production and practices, resistance (return adaptation) to the mutation of the state. Examples of the first model would have the calls of «surrounding the congress», «dignity marches» and «escraches»; as examples of the second «citizen's tides» are identified. In between you can find platforms that help the victims of the crisis (PAH).

Keywords: social movements, consumer culture, protest, political participation, civil society.

\section{Introducción}

Vista en perspectiva, la cultura del estado de bienestar se observó como fin máximo al que debe tender toda sociedad. Inscrita en las elaboraciones teóricas socioeconómicas de la modernización y el desarrollo, se intentó exportar el modelo occidental a todas las sociedades que no habían llegado a ese estadio ideal. Como toda idealización, exageraba los logros y oscurecía las causas profundas. Ocultaba la diferenciación de funciones que en la estructura mundial 
del capitalismo desempeñaban el centro desarrollado y la periferia en desarrollo. A pesar de la quiebra incipiente del estado de bienestar en los años 70, las ideas y presupuestos de la modernidad continúan hasta hoy en día en los discursos y prácticas de desarrollo.

La crisis global del capitalismo en los años 70 quebró este modelo de estado de bienestar. El diagnóstico económico fue de sentido contrario al de los años 30 del mismo siglo. La crisis no se debía a la ausencia de capacidad de consumo de la sociedad, sino todo lo contrario: el incremento de los costes de producción. El trabajo fue la principal variable productiva a modificar: flexibilización, segmentación, contención de los salarios y de las prestaciones sociales previstas para las situaciones de inactividad por el desempleo o la jubilación. La noción de progreso de la civilización occidental también entró en crisis, no sólo por el deterioro de las condiciones de trabajo y la inseguridad, sino también por los límites medioambientales al crecimiento y por la conciencia de las consecuencias del crecimiento de las desigualdades entre naciones.

La salida a la crisis, bajo la promesa de un paréntesis (años 90 y 2000) para el regreso a los estándares del estado de bienestar, no resultó más que un espejismo. Las consecuencias derivadas de las políticas monetarias, y de la expansión del capitalismo financiero sin sustento en la capacidad productiva y la economía real, han despejado las dudas. Al final del camino no había nada. Los indicadores socioeconómicos (pobreza, desigualdad, desempleo) lo manifiestan claramente y la protesta de las sociedades del sur de Europa es el efecto del choque de realidad. Es un punto de inflexión evidente, cuya profundidad y transcendencia se aprecia progresivamente. Así, «la crisis económica de 2007 y el hecho de que en España alcanzara su punto álgido en 2011, ha supuesto un punto y aparte en la mayoría de las tendencias no tecnológicas o relacionadas con la sociedad de la información. En ese sentido, cabe hablar de una situación disruptiva, donde el futuro ya no puede ser una deducción lógica efectuada sobre las tendencias dominantes en el pasado» (Alaminos, 2013: 16).

No obstante, en España, en este camino se fue formando una cultura del bienestar. El estado de bienestar y la sociedad de consumo están muy vinculados. El acceso de la clase trabajadora a multitud de bienes ociosos (Ortí, 1994) permitió la formación de una sociedad de consumo donde los miembros de la familia ya no se observan como unidades de producción (como acontecía en el medio rural e industrial), sino como unidades de consumo. Alonso asocia las ideas de integración económica de las clases trabajadoras del fordismo con el surgimiento de la cultura del consumo. Una cultura del consumo que, si bien ha mutado en la etapa postfordista (tras la crisis de los años 80 y el fin de la guerra fría) hacia una mayor segmentación de productos y consumidores, no 
deja de tener importancia en el contexto actual. La figura del «nuevo consumidor» más pragmático, responsable e informado no deja de ser un individuo que construye su identidad a partir de estilos de vida que toma como signos externos de pertenencia los objetos que consume y que no es ni absolutamente dominado ni absolutamente libre. En la vida cotidiana coinciden consumo y otros ámbitos, y el consumo también puede ser el marco de creación de nuevos movimientos sociales: «el proceso de consumo está incrustado en todos los mecanismos de funcionamiento de la vida, y no sólo del mercado; tampoco puede ser el agujero negro que absorba todas las riquezas y energías sociales. Bienestar, educación, salud y consumo no son elementos aislados que coinciden sólo en las mentes de los teóricos, son facetas de la ciudadanía misma en todas sus dimensiones» (Alonso, 2006: 333).

Uno de los principales atributos de la cultura del consumo es la reflexividad. Es decir, la capacidad de la sociedad de observarse a sí misma a partir de las representaciones, visiones que marcan formas de conducta y prácticas sociales. En la cultura del consumo, los principales agentes de reflexividad han sido los medios de comunicación. Obviando la publicidad como el contenido menos sutil, los contenidos mediáticos, tanto informativos como de entretenimiento o ficción, han facilitado el suministro de estas representaciones no sólo a partir de la exposición de ídolos mediáticos, sino también mostrando lo cotidiano y los acontecimientos excepcionales como el 15M. Algo de esto ocurrió en el 15M, cuya reivindicación primaria (que conjuga la doble identidad de consumidores y ciudadanos; consumidores responsables e informados y ciudadanos con derechos políticos) se observa en la génesis de la protesta contra los efectos y la gestión de la crisis. La plataforma de internet DRY tuvo en uno de sus promotores al grupo reivindicativo «No les votes», que surgió a partir de la protesta por parte de partidarios del conocimiento libre y de simples consumidores de contenidos por Internet a raíz de las revelaciones de wikileaks. Éstas mostraban las presiones norteamericanas (de los lobbys de su industria cultural) para un cambio legislativo dirigido a la ilegalización de los archivos compartidos en Internet; cambio que se materializó con la aprobación de la denominada ley Sinde (apellido de la ministra de cultura que redactó la ley). Bajo el nombre de «No les votes» los activistas integrados en esta asociación propugnaba orientar el voto hacia cualquier partido minoritario como protesta contra los partidos mayoritarios que habían firmado la ley. Es importante señalar esta doble identificación originaria de los participantes en la protesta: como ciudadanos (agentes políticos con derechos, libertades y expresión política) y como consumidores: de objetos de consumo y de medios (TV y redes sociales), así como de contenidos de la industria cultural (música y cine, principalmen- 
te). Los sentimientos de pertenencia ya fueron mutando con anterioridad a este escenario de crisis, intensificados en la época de crecimiento y más presente en las generaciones jóvenes, donde la movilidad geográfica y la inestabilidad laboral producen la sensación de transitorialidad:

La identificación se desarrolla preferentemente en el ámbito del consumo y no en el de la producción. La identificación con el ámbito profesional, por razones de desarrollar el mismo tipo de trabajo, y por clase social, es prácticamente inexistente. En ese sentido, las transformaciones del sistema capitalista han logrado en la España del siglo XXI girar las identificaciones desde el espacio de la producción, donde el capital es, en cierto modo, el elemento dependiente; hacia el espacio del consumo, donde lo es el trabajador. La disolución del sujeto social (clase social, trabajo) asocia lógicamente la desaparición de la identificación con las ideologías que hablan de él. La búsqueda de anclajes de identidad ha encontrado en las ofertas del mercado de consumo un repertorio, al parecer, válido (Alaminos y Penalva, 2010: 787).

Por tanto, la vertiente identitaria de la protesta ante la crisis va más allá del consumo, la subsistencia y las estrategias particulares. El resultado del desarrollo de las políticas de bienestar es la formación de una cultura del bienestar. Esta supuso cambios en las prácticas, pero también en la mentalidad. La cultura del bienestar opulenta asociada al consumismo es una cultura inconsciente: se disfruta del momento, sin preocuparse de las raíces de esta seguridad y del despliegue del consumo. Es ahora cuando los recortes en los derechos sociales van provocando la sensación de añoranza de tiempos pasados mejores, cuando el estado de bienestar se perfila mejor en su forma identitaria (vincula internamente a las clases medias en retroceso y a las clases trabajadoras, y diferencia estas sociedades de otras que no lo han disfrutado y que nunca las disfrutarán) a la que no se puede renunciar. Es por eso que ha producido la idea de que la asistencia del Estado y la igualdad de oportunidades no es producto de un acto de caridad o algo caprichoso, sino un derecho ciudadano (a través de impuestos y a través de contribuciones detraídas del salario) conseguido a partir de una serie de luchas históricas por la justicia social, y con el fin de asegurar un conjunto más o menos definido de valores y fines colectivos.

En este contexto de crisis, la autoubicación en el sistema jerárquico de estatus, es decir, la percepción subjetiva de clase social, refleja esta pérdida. En la escala del 1 al 10 entre los años 2009 a 2012, la media de estatus en España descendió del 6 al 5 (Alaminos y Penalva, 2013), donde el significado de autoubicarse en la clase media va asociado, entre otros aspectos, a la capacidad de consumo de bienes ociosos. Por otro lado, las expectativas de ascenso social generadas con anterioridad no tienen continuidad. La sociedad española 
piensa que vive mejor que las generaciones de sus padres, al mismo tiempo que observa el futuro de la generación de sus hijos con pesimismo (Alaminos, Penalva, Domenech, 2014 b). La crisis económica y sus efectos sobre la pérdida de estatus, el incremento de las desigualdades y la pobreza, así como la mala gestión política de la misma, han producido una presencia continua de protesta de niveles no conocidos desde que existen estadísticas.

\section{Marco teórico}

Las elaboraciones teóricas que han sido revisadas y que constituyen el marco de interpretación de la protesta en España provienen de dos campos de investigación. En primer lugar, de las investigaciones relativas a la cultura del consumo como fuente de identidad que han sido sistematizadas por Alonso (2006), con referencias a la centralidad y grupalidad del consumo, a la configuración de una sociedad reflexiva (Beck, Giddens, Lash, 1994) y a la base productiva y reproductiva del modelo postfordista. En segundo lugar, a las provenientes de la teoría de los movimientos sociales y la acción colectiva, tanto las que hacen referencia a los condicionamientos estructurales y coyunturales de los conflictos políticos (Tilly, 2005; McAdam, 1996; McAdam, Tarrow y Tilly, 2001), como las que analizan las formas que adoptan estos movimientos en la discusión conceptual entre nuevos y viejos (Gunder Frank y Fuentes, 1987; Melucci, 1994; Touraine, 1997), así como la incorporación al análisis del elemento identitario y las condiciones de quiebra de la cotidianidad como principios de movilización espontánea (Piven y Cloward, 1997). Se tienen en cuenta, además, las aportaciones que dan importancia a las emociones en la acción política (Jasper, 2012) y a las nuevas formas de acción colectiva que incluyen la vertiente política del consumo (Micheletti y McFarland, 2010).

\section{Metodología y fuentes de datos}

El estudio parte de una metodología cualitativa, pero con referencias cuantitativas relativas a datos estadísticos que sirven como elementos para la contextualización (datos socioeconómicos) y para la problematización del estudio (coincidencia temporal de la crisis económica y social, crisis del estado del bienestar e incremento de la protesta). Las fuentes de datos son secundarias, a partir de la lectura de textos sobre la movilización y la protesta en España, el análisis de los manifiestos provenientes de los movimientos sociales y textos provenientes de 
los medios de comunicación. No tiene sentido el muestreo probabilístico. Los textos relativos a los manifiestos han sido seleccionados de forma intencional, abarcando la diversidad de discursos que la movilización de la protesta ha producido desde 2011 hasta 2014: desde el manifiesto de DRY de mayo de 2011 a los manifiestos de movimientos y acciones de protesta posteriores a estas fechas: mareas, PAH, marchas por la dignidad, Plataforma en Pie.

\section{Análisis}

El incremento incesante del desempleo y la consecuente caída estrepitosa del consumo de bienes ociosos desde el máximo del periodo de expansión (alcanzado en 2007 y que se constituye como fuente de estatus en las sociedades de masas), coinciden temporalmente con el incremento de la protesta. Sin dejar de lado la dificultad creciente durante los años de la crisis para la adquisición de bienes básicos relacionados con la subsistencia de una proporción significativa de la población (en 2013 un 28,8 \% de la población estaba en riesgo de exclusión social), se observa que en 2010 decae el consumo de elementos identitarios vinculados a la moda -como son los artículos de ropa y vestido-y, más evidentes, los automóviles.

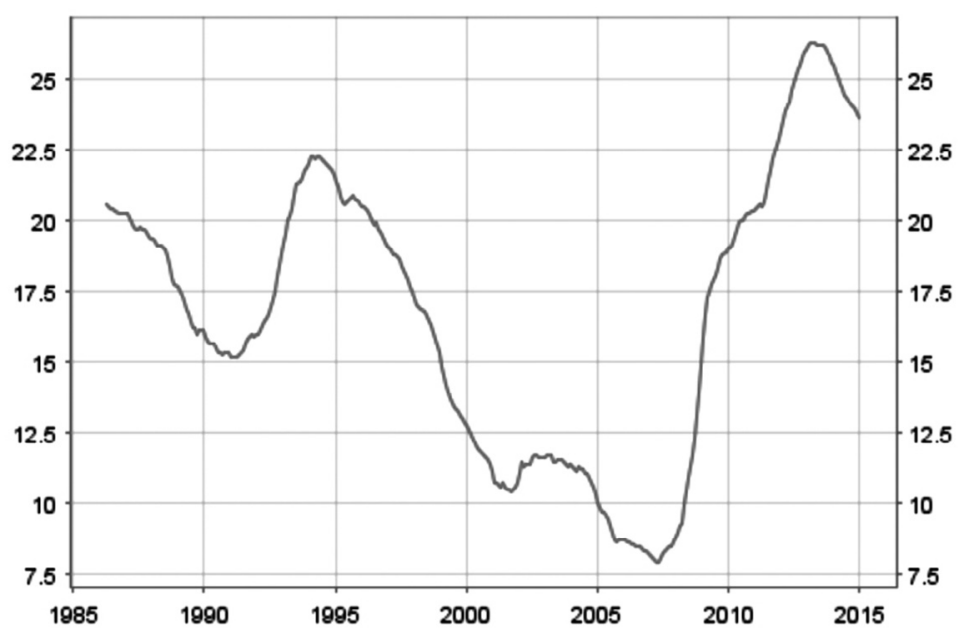

Fuente: BCE (http://sdw.ecb.europa.eu/quickview.do?SERIES_KEY=132.STS.M.ES.S.UNEH.RTT000.4.000)

Gráfico 1. Tasa de desempleo en España (1985-2015) 


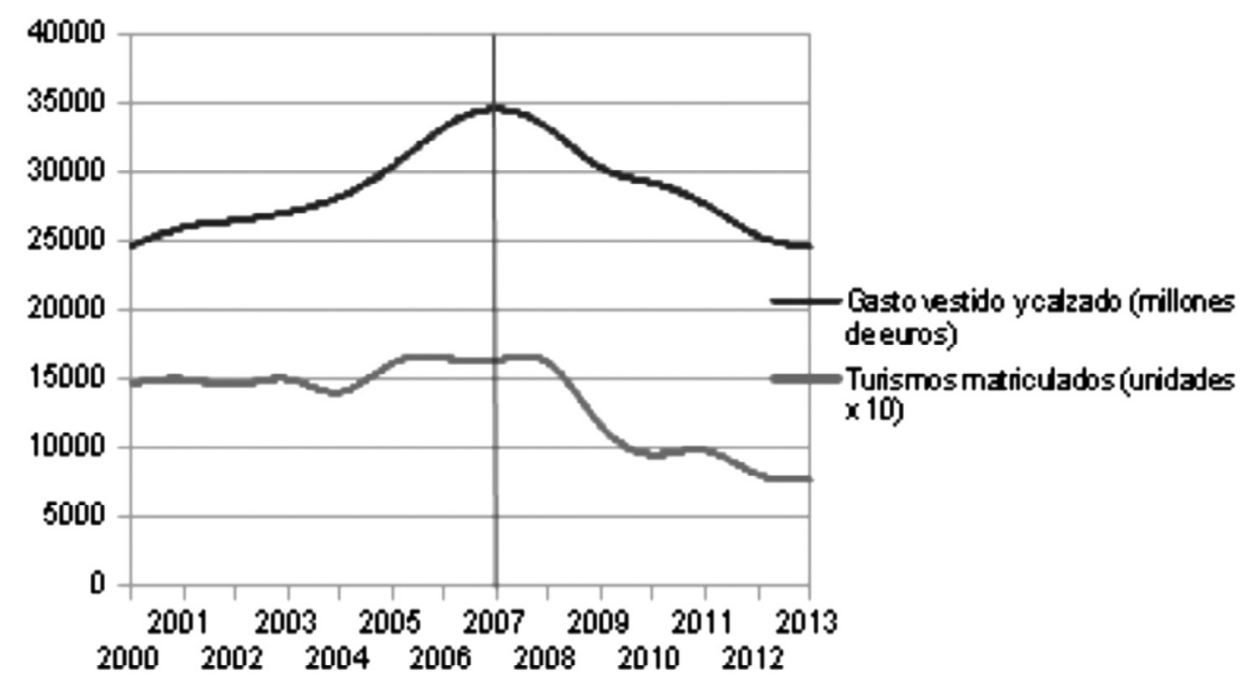

Fuente: INE (encuesta de condiciones de vida y encuesta de presupuestos familiares) y ANFAC

Gráfico 2. Gasto en ropa y calzado y en automóviles (2000-2013)

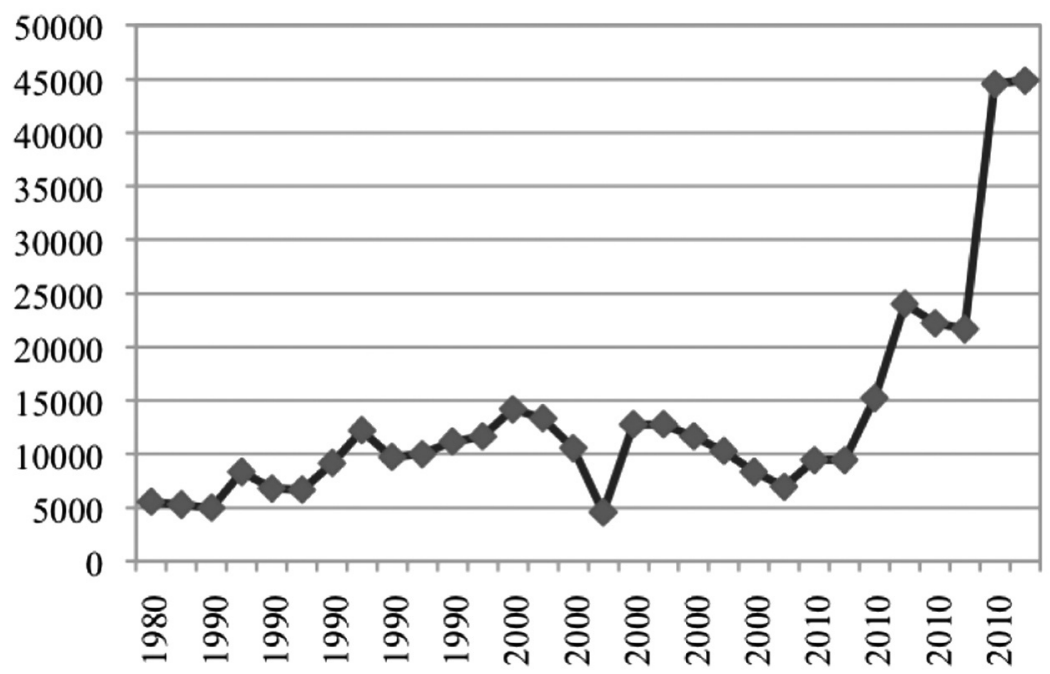

Fuente: elaboración propia, a partir de Jiménez Sánchez (2011) y anuarios del Ministerio de Interior (varios años)

Gráfico 3. Número de manifestaciones en España (1984-2013) 
El 15M muestra un punto de inflexión en las representaciones políticas y actitudes hacia lo público en la sociedad española desde la transición democrática de los años 70 y 80 . El mismo nombre de la plataforma impulsora de este movimiento refleja el contenido de la misma: Democracia Real Ya. Por un lado supone el «redescubrimiento» de la democracia, una democracia que se desea rescatar de la acción desgraciada de los representantes políticos, puesto que los gobernantes y la mayoría de los parlamentarios sirven al interés de una elite (indefinida pero que marca las reglas del juego para el beneficio del sector financiero y de las grandes empresas, a los cuales se culpa de la crisis). No son autónomos (se pierde soberanía por las imposiciones foráneas de la troika: Comisión Europea, Banco Central Europeo y FMI) y tienden a realizar prácticas ilegales (corrupción alimentada principalmente por la burbuja especulativa consustancial al desarrollo del sector inmobiliario) y legales (reformas legislativas y laborales que conllevan privatizaciones, reducción de derechos y libertades y desmantelamiento del estado de bienestar), pero que son consideradas ilegítimas porque perjudican al conjunto de la sociedad en beneficio de una minoría. Se concentra la crítica fundamentalmente en los dos principales partidos que se han alternado en el gobierno desde la transición: PP y PSOE. Los lemas del 15M son «no les votes», como forma de acabar con el bipartidismo, y «no nos representan», en el sentido de representar intereses ajenos a la gran mayoría de los votantes y de incumplir las promesas electorales. Por otro lado, la inmediatez; el «ya» indica el presente o el futuro más cercano, implica acción y exigencia. No se debe esperar a un momento electoral porque la democracia es mucho más que la cita con las urnas. La democracia es lo que se está haciendo: ejercer el derecho a la protesta, ocupar el espacio público y convencer a la mayoría de la población para que se una al movimiento. El término «régimen» utilizado en algunos manifiestos permiten la identificación del movimiento con los precedentes del norte de África, algunos de los cuales produjeron rupturas institucionales.

Ante esta situación, y mediante la toma espontánea de la calle y de las plazas, se intenta llevar a cabo prácticas políticas que sí son consideradas propias de una verdadera democracia: horizontalidad y toma de decisiones tras un debate abierto mediante asambleas. Se trata del regreso al ágora griega (discusión pública en la plaza sobre asuntos públicos por parte de sujetos libres e iguales). Lo que se reivindica con la propia acción es la autonomía («si no nos representan, nos presentamos a nosotros mismos») y la capacidad de construir propuestas orientadas a un nivel superior generadas desde abajo. Supone autonomía y autogestión, pero también interés y conocimiento de los 
asuntos públicos. Tras décadas de cierta atonía de la participación política y del interés por la política, el $15 \mathrm{M}$ ha despertado la democracia tanto en sentido práctico (reflexión sobre la forma de organizarse y de tomar decisiones y su actuación consecuente) como de conocimiento de los asuntos cívicos (derechos y libertades, economía política -deuda, valor, desigualdad-) y de los efectos de la globalización sobre la vida cotidiana, el medio ambiente y las relaciones humanas. Ambos aspectos relacionan lo institucional con lo cotidiano, presentando un ejercicio de aprendizaje político.

La protesta, junto con este primer efecto de aprendizaje político, tiene un segundo efecto sociopolítico, que es el del fortalecimiento de la sociedad civil. Aunque no está expresado explícitamente por parte de los protagonistas de la protesta, el movimiento deja de lado, o incluso supera, el conflictivo concepto de tercer sector-que media entre el Estado y el mercado, pero que suple las carencias del primero para intentar alcanzar la universalidad de la protección social y que facilita la incorporación del segundo en una extraña mezcla de voluntarismo y negocio- para recuperar un concepto tan consustancial a la democracia como es el de sociedad civil. En términos sociopolíticos, la sociedad civil es el reino de la autonomía de la sociedad frente al poder coercitivo del Estado, el ámbito del interés común frente al beneficio individualista competitivo del mercado y el lugar de la libre asociación donde se dirimen los conflictos ideológicos por la hegemonía política y cultural. En estos años de crisis y protestas, la movilización, por otro lado, ha desarrollado la ayuda mutua y ha generado unos vínculos entre sus participantes que ha hecho desarrollar un conjunto de iniciativas de asistencia a los más afectados por la crisis, de prácticas económicas comunitarias (intercambios de tiempo, bienes y servicios; economía colaborativa; monedas sociales) (Alaminos, Penalva y Domenech, $2014 a$ ) que han supuesto un incremento del capital social, entendido como extensión de redes de apoyo (Putnam, 1993).

Es tal el efecto conjunto de crisis y movilización sobre la sociedad española que, observado de forma diacrónica, se visualiza el incremento de la implicación política en términos de trabajo asociativo. 


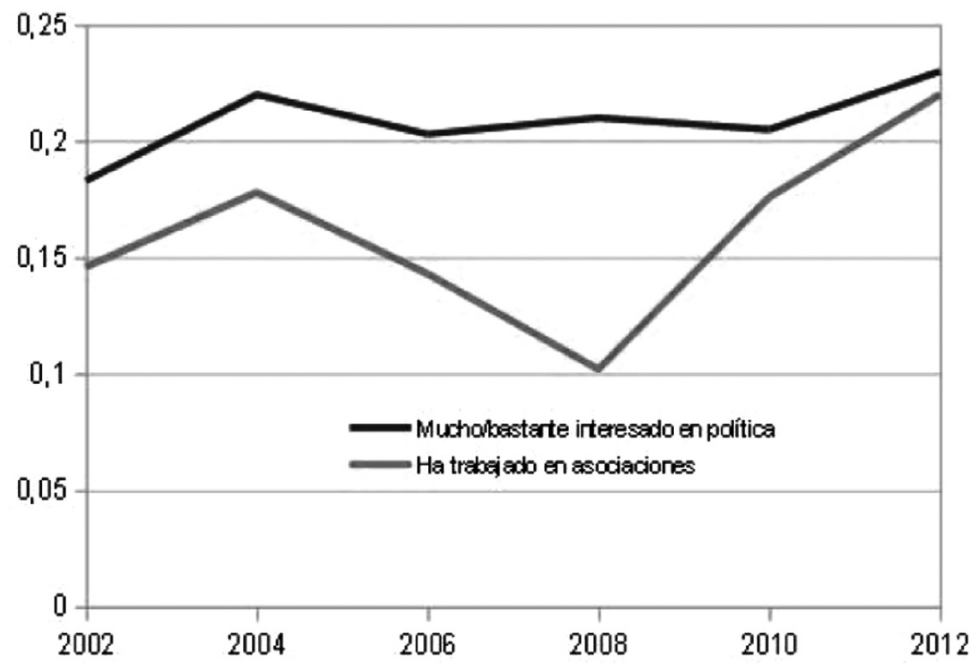

Fuente: elaboración propia a partir de los datos de la Encuesta Social Europea (2000-1012): http:// www.europeansocialsurvey.org/downloadwizard/

Gráfico 4. Interés por la política y trabajo en asociaciones (España, 2002-2012)

Pero dada la heterogeneidad del movimiento, no todo son experiencias nuevas y aprendizaje primario; en el $15 \mathrm{M}$ convergen movimientos sociales que tienen una existencia y experiencia previas en la acción colectiva. Los movimientos herederos de las reivindicaciones altermundistas, que denunciaban las enormes desigualdades generadas por el capitalismo global y sus nefastas consecuencias sobre el medioambiente y la pobreza, como los movimientos pacifistas, ecologistas, anticapitalistas, feministas que tuvieron su protagonismo en la oposición a la II Guerra del Golfo en 2003, se incorporaron desde los inicios al movimiento. Es la doble alma del movimiento que señala Taibo (2011): críticos anticapitalistas, por un lado, y jóvenes desempleados y precarios que expresan su indignación ante la falta de expectativas de futuro. Con diferente papel en el movimiento, consiguen coexistir y dar impulso a la protesta. La ausencia de organizaciones políticas tradicionales es patente y el movimiento no se deja instrumentalizar por ningún partido político o sindicato. De hecho, los símbolos que identificaron estos «viejos movimientos sociales» son censurados; las precarias estructuras incipientes son horizontales y se evita la emergencia de líderes y portavoces con jerarquía orgánica. Esta dualidad, 
en el marco del estudio de la participación política no convencional, se ve completada con la conjugación de la vertiente expresiva de los movimientos (acciones políticas conducentes a la manifestación de un descontento) con la instrumental (acciones orientadas al cambio político y social).

Con el tiempo, el hecho de que las causas de fondo de la protesta no hayan desaparecido (crisis económica y política), sino que se hayan agravado (indicadores recientes de desigualdad y pobreza son muestras evidentes del desmantelamiento del estado de bienestar, precisamente en el momento en que más se necesita, y existe un mayor conocimiento de las prácticas de corrupción legales e ilegales experimentadas antes y durante la crisis), la protesta no sólo no ha cesado, sino que se ha incrementado. Lo que ocurre es que el movimiento se transforma (como indica su propio nombre, cuando un movimiento comienza a dar muestras de parálisis, deja de ser tal para convertirse en otra cosa) y observamos dos herencias del $15 \mathrm{M}$ en cuanto a las formas y reivindicaciones políticas: a) un tipo de participación política que supone una continuación $15 \mathrm{M}$ con un mayor peso del componente expresivo y emocional de la acción política (como muestran algunos lemas de la protesta en sus inicios: «Gobierno del pueblo para el pueblo», «Otra forma de hacer política es posible», «Política = amantes de dinero», «Yo no soy anti-sistema, el sistema es anti-yo», «si no nos dejan soñar, no les dejaremos dormir») que tiene como marco de producción de discursos y prácticas la idea de transformación social hacia otro modelo productivo y medioambiental, de representación política, de cambio cultural; y b) un tipo de participación política (con un mayor peso del componente racional, cognitivo) que tiene como marco de producción de discursos y prácticas la resistencia (retorno, adaptación) frente a la mutación del Estado. Ambos podrían dibujar una secuencia en el tiempo, provocada respectivamente por los efectos de dos choques emocionales producidos por la crisis del desempleo (2010) y la crisis del estado del bienestar (2012-14), respectivamente, pero que empíricamente están entrelazados. Es común encontrar individuos que participan activamente en diferentes asociaciones y organizaciones de ambos tipos, lo que ha proporcionado el intercambio de experiencias, estrategias, marcos interpretativos y principios ideológicos que sirven de orientación para la acción política.

\section{Conclusiones}

El análisis de la protesta desarrollada en los últimos años en España nos muestra una activación de la participación política. Esta participación ha 
permitido fortalecer la sociedad civil a partir de una red de organizaciones muy heterogéneas (aquí se ha pretendido establecer una tipología basada en las formas de la protesta) conectadas entre sí, teniendo como elemento común la denuncia de la situación de crisis social y la ineficiencia de la gestión política. Desde esta perspectiva, la evolución de los movimientos sociales surgidos o reactivados tras el $15 \mathrm{M}$ ha supuesto un aprendizaje político para ciertos sectores que habían estado alejados del interés por la política y que tendrá efectos sobre el comportamiento político futuro de este país. En ese sentido, supone un periodo temporal en el cual la socialización política de las generaciones más jóvenes se ha llevado a cabo de forma más intensa. Los datos para España de la Encuesta Social Europea muestran la tendencia de incremento del interés por la política y la participación asociativa, en contraste con periodos anteriores.

Con respecto al análisis del proceso de la protesta y la configuración de los movimientos sociales, se plantean líneas de investigación que tendrán como objetivo el estudio de los componentes expresivo, instrumental, racional y emotivo de la participación en los movimientos desde una perspectiva etnográfica. El estudio de los datos secundarios (manifiestos) puede limitar el establecimiento de conclusiones definitivas sobre la motivación y experiencia de los actores sociales y la percepción de la población en general sobre la predisposición al cambio social. Una investigación cualitativa a partir de técnicas basadas en la conversación podría dar luz sobre cómo se relacionan los discursos sobre la participación política y su vinculación con lo cotidiano, incluidas las cuestiones identitarias vinculadas tanto con la identificación partidaria e ideológica, como con aspectos cotidianos tan presentes como es el consumo. Se puede iniciar, por tanto, una línea de trabajo que explore cómo se articulan las prácticas económicas comunitarias recuperadas en los tiempos de crisis (basadas en la autogestión y la solidaridad) con aspectos relacionados con el consumo responsable, aquél que incorpora valores relacionados con la igualdad y la conservación del medio ambiente. Ciertas propuestas tienen capacidad de extenderse socialmente tras las experiencias de movilización social surgidas en estos años de crisis. Entre muchos otros, tenemos ejemplos de propuestas que desarrollan los movimientos que abogan por un cambio de modelo productivo y que conjugan discursivamente las críticas a las grandes empresas, que concentran gran parte de la producción y distribución de bienes y servicios, con la valoración del comercio y la producción locales y comunitarios. 


\section{Referencias bibliográficas}

Alaminos, A. (2013): "La futura vida cotidiana en España: la sociedad de consumo», OBETS. Revista de Ciencias Sociales, 8 (1): 15-57.

Alaminos, A.; C. Penalva (2010): «La vida cotidiana en la España del siglo XXI» en Campo, S.; J. F. Tezanos (coords.): España: una sociedad en cambio, Madrid, Biblioteca Nueva, 759-809.

- (2013): «España: de los impactos de la crisis a las movilizaciones de protesta», Ecuador Debate, 59: 93-118.

Alaminos, A.; C. Penalva; Y. Doménech (2014 a): «Reacciones comunitarias a la crisis económica y social en España», AZARBE-Revista Internacional de Trabajo Social y Bienestar, 3: 47-53.

- (2014 b): «A mobilidade social intergeracional subjetiva e as expectativas em relação ao futuro. Brasil, Rússia, Índia em comparação com Espanha, Itália e Grã-Bretanha», $2^{\circ}$ Encontro Internacional de Política Social, UfES, Brasil, 4-7 de agosto, 2014.

Alonso, L. E. (2006): "Consumo y cultura de consumo en el marco de la fragmentación social: visiones y tendencias» en Bericat, E. (coord.): El cambio social en España: visiones y retos de futuro, Sevilla, Centro de Estudios Andaluces, Junta de Andalucía, 313-338.

BecK, U.; A. Giddens; S. LASH (eds.) (1994): The Reflexive Modernization. Politics, Tradition and Aesthetics in the Modern Social Order, Stanford, Standford University Press.

JASPER, J. (2012): «¿De la estructura a la acción? La teoría de los movimientos sociales después de los grandes paradigmas», Sociológica, 27: 7-48.

Jiménez SÁnchez, M. (2011): «La normalización de la protesta. El caso de las manifestaciones en España (1980-2008)», Opiniones y Actitudes, 70, Madrid, CIS.

McAdaM, D. S. (1996): «Conceptual Origins, Current Problems, Future Directions», en McAdam, D.; J. D. McCarthy; M. N. Zald (eds.): Comparative Perspectives on Social Movements, Cambridge, Cambridge University Press, 23-40.

McAdaM, D. S.; S. TARrow; C. Tilly (2001): The Dynamics of Contention, Cambridge, Cambridge University Press.

MeluCCI, A. (1994): «¿Qué hay de nuevo en los nuevos movimientos sociales?» en Gusfield, J.; E. Laraña (coords.): Los nuevos movimientos sociales: de la ideología a la identidad, Madrid, CIS, 119-150.

Micheletti, M.; A. MCFarland (2010) (eds.): Creative Participation: Responsibility-taking in the Political World, Boulder, Paradigm Publischers. 
OrTí, A. (1994): «La estrategia de la oferta en la sociedad neocapitalista de consumo: génesis y praxis de la investigación motivacional de la demanda», Política y Sociedad, 16: 37-92.

Piven, F. F.; R. Cloward (1979): Poor People's Movements: Why They Succeed, how They Fail, New York, Vintage Books.

PutnaM, R. D. (1993): «The Prosperous Community: Social Capital and Public Life», The American Prospect, 13: 35-42.

Taibo, C. (2011): Nada será como antes. Sobre el movimiento 15M, Madrid, Catarata.

Touraine, A. (1997): ¿Podremos vivir juntos? La discusión pendiente: el destino del hombre en la aldea global, Buenos Aires, Fondo de Cultura Económica. 